\title{
Activated Carbon from the Chinese Herbal Medicine Waste by $\mathrm{H}_{3} \mathrm{PO}_{4}$ Activation
}

\author{
Tie Mi, Liang Chen, Shan-zhi Xin, and Xin-ming Yu \\ Hubei Key Laboratory of Industrial Fume and Dust Pollution Control, School of Chemical and Environmental Engineering, \\ Jianghan University, Wuhan 430056, China
}

Correspondence should be addressed to Tie Mi; mitie1999@163.com

Received 7 August 2015; Revised 9 October 2015; Accepted 11 October 2015

Academic Editor: Pingmei Guo

Copyright (c) 2015 Tie Mi et al. This is an open access article distributed under the Creative Commons Attribution License, which permits unrestricted use, distribution, and reproduction in any medium, provided the original work is properly cited.

Large amounts of Chinese herbal medicine wastes produced by the medicinal factories have been mainly landfilled as waste. In this study, via phosphoric acid activation, a Chinese herbal medicine waste from Magnolia officinalis (CHMW-MO) was prepared for activated carbon (CHMW-MO-AC). The effect of preparation conditions (phosphoric acid/CHMW-MO impregnation ratio, activation temperature, and time) of activated carbon on yield of CHMW-MO-AC was investigated. The surface area and porous texture of the CHMW-MO-ACs were characterized by nitrogen adsorption at $77 \mathrm{~K}$. The $S_{\mathrm{BET}}$ and pore volume were achieved in their highest value of $920 \mathrm{~m}^{2} / \mathrm{g}$ and $0.703 \mathrm{~cm}^{3} / \mathrm{g}$, respectively. Thermal gravity analysis and scanning electron microscope images showed that CHMW-MO-ACs have a high thermal resistance and pore development. The results indicated that CHMW-MO is a good precursor material for preparing activated carbon, and CHMW-MO-AC with well-developed mesopore volume can be prepared by $\mathrm{H}_{3} \mathrm{PO}_{4}$ activation.

\section{Introduction}

Large amounts of Chinese herbal medicine wastes (CHMWs) produced as by-products in pharmaceutical process of traditional Chinese crude medicines cause serious solid waste disposal problems in China. Due to absence of proper disposal methods, CHMWs are mainly landfilled or optionally disposed as solid wastes. So, CHMWs were proposed for composting by some researchers, but CHMWs are poor in nutrient as fertilizer but abundant in cellulose, hemicellulose, and lignin, which are usually difficult to digest or degrade in composting [1]. Therefore, it is deadly required to provide a cost-effective, high value-added, and innovative solution to the problems caused by CHMWs.

For CHMWs, a possible utilization is converting it into activated carbon. Although activated carbon prepared from Agro-industrial by-products has been investigated, there is no systematic research regarding the activated carbon prepared from CHMWs in the current literatures. For its feasibility study on preparing activated carbon, the CHMW of Magnolia officinalis (MO) (denoted by CHMW-MO) as a precursor for a low-cost activated carbon preparation was investigated in this paper. Because of its one-step simple process, lower activation temperature, higher yield, shorter activation time, and higher development of porosity [2, 3], phosphoric acid activation has been widely recognized for production of activated carbon from lignocellulosic materials. So, in the paper, phosphoric acid was used as activating agent for preparing activated carbon from CHMW-MO. And the effects of phosphoric acid concentration, activation temperature, and impregnation ratio and activation time on yield of CHMW-MO-AC were studied. The results showed this is a promising approach for the activated carbon preparation.

\section{Materials and Methods}

2.1. Materials. The Chinese herbal medicine wastes Magnolia officinalis (CHMW-MO) was collected from Li Shi-Zhen Pharmaceutical Group Limited, China. It was dried in sunlight until 15\% water content was reached. The dried material was crushed, sieved for desired particle size $(<1.7 \mathrm{~mm})$, and used for preparation of activated carbon. Phosphoric acid 
TABLE 1: The amount of PA acid prepared for three different concentrations.

\begin{tabular}{cccc}
\hline PAC & \multicolumn{3}{c}{ IR } \\
& 1.5 & 2.5 & 3.5 \\
\hline \multirow{2}{*}{$30 \%$} & PA:water & PA $:$ water & PA $:$ water \\
& $17.64 \mathrm{~g}: 32.36 \mathrm{~g}$ & $29.41 \mathrm{~g}: 53.92 \mathrm{~g}$ & $41.18 \mathrm{~g}: 75.5$ \\
\hline \multirow{2}{*}{$50 \%$} & PA:water & PA $:$ water & PA: water \\
& $17.64 \mathrm{~g}: 12.36 \mathrm{~g}$ & $29.41 \mathrm{~g}: 20.59 \mathrm{~g}$ & $41.18 \mathrm{~g}: 28.82$ \\
\hline \multirow{2}{*}{$70 \%$} & PA:water & PA $:$ water & PA $:$ water \\
& $17.64 \mathrm{~g}: 3.83 \mathrm{~g}$ & $29.41 \mathrm{~g}: 6.3 \mathrm{~g}$ & $41.18 \mathrm{~g}: 8.82$ \\
\hline
\end{tabular}

(PA) (analytical reagent (AR), $85 \mathrm{wt} \%$ ) was obtained from Soochow Weixu Trading Co., Ltd, China.

2.2. Preparation of Activated Carbon. On the basis of phosphoric acid and three different impregnation ratios, three different phosphoric acid concentrations (PAC) of 30\%, $50 \%$, and $70 \%$ were prepared, respectively. The amount of phosphoric acid was shown in Table 1. Impregnation ratio (IR) is defined as the weight ratio of pure phosphoric acid and dried CHMWs which was 1.5, 2.5, and 3.5, respectively. A certain amount $(10 \mathrm{~g})$ of the dried CHMW-MO was soaked in three different concentration phosphoric acid solutions, respectively, and then the mixture was continuously shaken and stirred for 12 hours to ensure the access of phosphoric acid to the interior of the CHMW-MO.

After being filtered, the mixture was dehydrated at $105^{\circ} \mathrm{C}$ in the oven overnight until constant weight of the mixture was reached; then it was carbonized and activated simultaneously inside a completely sealed horizontal quartz tube reactor (40 millimeters in the diameter and 1500 millimeters in the height) under high purity nitrogen (99.99\%) flow of $120 \mathrm{~mL} \mathrm{~min}^{-1}$, at final temperatures in the range of $350-550^{\circ} \mathrm{C}$ and activation time for 35,50 , and 65 minutes, respectively. Once activation time was reached, heating was stopped, and then the gas flow was continued to cool quartz tube down to room temperature. After cooling, the activated product was taken out and washed with deionized water to remove remaining chemical until the washed solution became neutral. The activated carbon was dried at $105^{\circ} \mathrm{C}$ for $12 \mathrm{~h}$. Subsequently, the activated carbon was weighted and stored in plastic containers for further studies. All experiments were repeated 3 times. The yield of the CHMWMO-AC is defined as the ratio of the weight of the final activated carbon $W$ to that of the dried CHMW-MO $\left(W_{0}\right)$ :

$$
\text { yield } \%=\frac{w}{w_{0}} \times 100 \% \text {. }
$$

2.3. Characterization of Activated Carbons. For an evaluation on thermal behaviors of MO, CHMW-MO, and CHMWMO-AC prepared at different impregnation ratios, A Netzsch STA-409 analyzer was used to perform their thermal gravity analysis (TGA) behavior characteristics. About $10 \mathrm{mg}$ of the sample was heated to a final temperature of $900^{\circ} \mathrm{C}$ at a heating rate of $10^{\circ} \mathrm{C} \mathrm{min}^{-1}$ under nitrogen gas flow to ensure an inert atmosphere in the system. Low heating rate of $10 \mathrm{~K} \mathrm{~min}^{-1}$
TABLE 2: Proximate, ultimate, and component analyses.

(a)

\begin{tabular}{lc}
\hline Proximate analysis of CHMW-MO (as received, wt \%) \\
\hline Moisture & 9.50 \\
Volatile matter & 56.3 \\
Fixed carbon & 27.36 \\
\hline
\end{tabular}

(b)

\begin{tabular}{lc}
\hline Ultimate analysis of CHMW-MO (dry basis, wt \%) & \\
\hline $\mathrm{C}$ & 48.51 \\
$\mathrm{H}$ & 5.9 \\
$\mathrm{O}^{*}$ & 43.89 \\
$\mathrm{~N}$ & 1.3 \\
$\mathrm{~S}$ & 0.4 \\
\hline
\end{tabular}

(c)

\begin{tabular}{lcc}
\hline \multicolumn{3}{c}{ Component analyses (dry basis, wt \%) } \\
& MO & CHMW-MO \\
\hline Cellulose & 26.78 & 25.48 \\
Hemicellulose & 20.24 & 18.03 \\
Lignin & 19.87 & 27.11 \\
\hline Ash & 6.20 & 2.84 \\
\hline
\end{tabular}

Note: ${ }^{*}$ obtained by difference.

was used in the test to minimize the temperature difference between the TGA chamber and sample and also maintain the sample for enough time at a specific temperature range so that perceptible thermal behavior could be obtained.

The BET surface area and pore size of CHMW-MO-ACs were determined by $\mathrm{N}_{2}$ adsorption at $77 \mathrm{~K}$ using a surface analyzer (Micromeritics, ASAP 2020). Prior to gas adsorption measurements, the carbon was degassed at $200^{\circ} \mathrm{C}$ in a vacuum condition for $24 \mathrm{~h}$. Nitrogen adsorption isotherms were measured over a relative pressure $\left(P / P_{o}\right)$ range from approximate 0.005 to 0.985 .

A sample morphology was observed using a scanning electron microscope (SEM, model JEOL 5400). The sample surface was coated with $\mathrm{Au}-\mathrm{Pd}$ to prevent electrical charging during examination. Imaging was done in the high vacuum mode under an accelerating voltage of $15 \mathrm{kV}$, using secondary electrons.

\section{Results and Discussions}

3.1. CHMW-MO Property. The $S_{\mathrm{BET}}$, proximate, and ultimate analyses of CHMW-MO are given in Table 2. The components of MO and CHMW-MO are also showed in Table 2.

The analytical results reveal that CHMW-MO has high carbon content approximately $48.51 \%$ and low ash content. These results indicate that CHMW-MO is suitable to be used as a precursor for activated carbon preparation, due to its low inorganic content and high carbon content. The $S_{\mathrm{BET}}$ of CHMW-MO was less than $3 \mathrm{~m}^{2} / \mathrm{g}$. Comparing the ash 


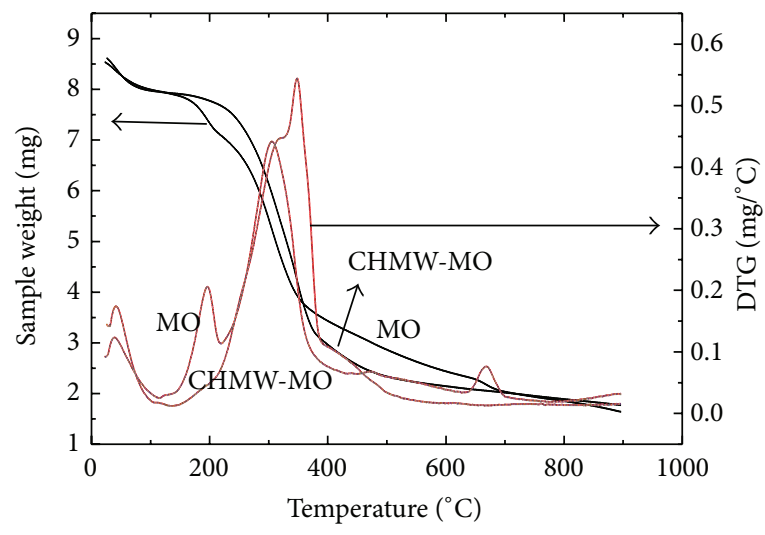

(a)

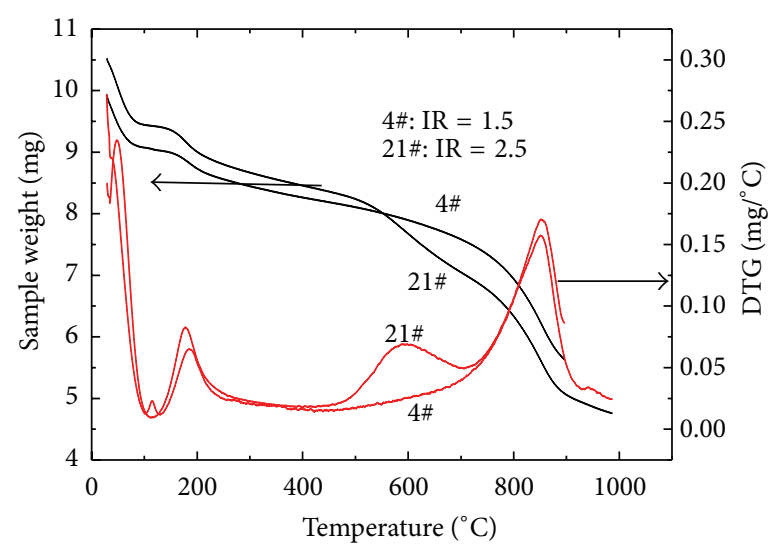

(b)

Figure 1: Thermogravimetric curves of different samples. (a) MO and CHMW-MO samples; (b) CHMW-MO-ACs with different temperatures.

content of CHMW-MO and MO, the ash content of CHMWMO sharply dropped from 6.20 to $2.84 \%$.

\subsection{Thermal Behavior Analyses of MO, CHMW-MO, and} CHMW-MO-ACs. Figure 1(a) shows weight losses of MO, CHMW-MO, expressed as weight $(w)$, and the derivative curve of the weight loss (DTG), against the pyrolysis temperature. $\mathrm{MO}$ and $\mathrm{CHMW}-\mathrm{MO}$ began to decompose at temperatures approximately $50-150^{\circ} \mathrm{C}$ (shown in Figure 1(a)), and the initial weight decreased due to the moisture evaporation. As observed, the second weight loss which is the major thermal degradation step takes place primarily in a relatively broad temperature interval, ranging approximately between 150 and $400^{\circ} \mathrm{C}$. The DTG curves of MO and CHMW-MO exhibited distinctly different weight loss peaks of thermal decomposition. Their thermal degradation might be attributed to the decomposition of the major biopolymers, namely, cellulose, hemicellulose, and lignin. For MO, the rate of weight loss rapidly rises above $150^{\circ} \mathrm{C}$ as hemicellulose decomposes; until approximately $200^{\circ} \mathrm{C}$ the rate of weight loss again increases and reaches a maximum at $300^{\circ} \mathrm{C}$, as the cellulose and lignin degrade. For CHMW-MO, the weightlessness peak at $150^{\circ} \mathrm{C}$ disappears; the largest weightlessness peak reaches $350^{\circ} \mathrm{C}$, at which the cellulose was largely decomposed. Above $400^{\circ} \mathrm{C}$, weight loss decreases gradually at considerably slower rates as the temperature was increased. The amount of solid residue at $900^{\circ} \mathrm{C}$ was $20 \mathrm{wt} \%$ of initial weight (approximately $10 \mathrm{mg}$ ), consisting of the carbonaceous residue or char. Similar results were reported by Cukierman et al. [4], and they suggested that the decomposition of biomass materials takes place through a complex network of reactions. Antal Jr. [5] investigated the thermal degradation of lignocellulosic material and found that the hemicellulose degrades in the temperature range $325-375^{\circ} \mathrm{C}$, while lignin degradation occurs more slowly over the wide temperature range $250-500^{\circ} \mathrm{C}$.

In order to detect the effect of the phosphoric acid impregnation ratio on the weight loss of activated carbon, weight losses of CHMW-MO-AC with different IR are illustrated in Figure 1(b). Compared with weight loss of
CHMW-MO in Figure 1(a), obviously different thermal degradation behavior was found for CHMW-MO-AC with different IR, as observed in Figure 1(b). In the first stage, the weight loss peak was observed between 50 and $180^{\circ} \mathrm{C}$ for the CHMW-MO-AC, due to moisture elimination [6] and pyrolysis of some residual organic matter [7]. Some researchers $[8,9]$ suggested that the most acidic oxygen-containing groups (carboxylic, phenolic) of synthetic chemically activated carbons begin to decompose at temperatures above $250^{\circ} \mathrm{C}$. For CHMW-MO-AC $(\mathrm{IR}=2.5)$, the peak observed at approximately $600^{\circ} \mathrm{C}$ might be attributed to acidic oxygencontaining groups pyrolysis. As the temperature continues to rise, great weight loss was produced in the temperature range between 700 and $900^{\circ} \mathrm{C}$. This observation could be attributed to the volatilization of phosphorus compounds $[10,11]$. According to Jagtoyen and Derbyshire [12], these phosphorus compounds include $\mathrm{H}_{3} \mathrm{PO}_{4}$ and $\mathrm{H}_{2} \mathrm{PO}_{4}{ }^{-1}$ or $\mathrm{H}_{2} \mathrm{P}_{2} \mathrm{O}_{7}{ }^{-2}$ which are eliminated from cellulose phosphate esters at temperatures over $450^{\circ} \mathrm{C}$. Puziy et al. [13] studied the changes of chemical composition with treatment temperature in the activated carbons prepared by phosphoric acid activation, and their results showed that carbonization of the polymer precursors in the presence of phosphoric acid resulted in the incorporation of phosphorus in the activated carbon, and the phosphorus content increases up to a level of about $9 \mathrm{wt} \%$ at $800^{\circ} \mathrm{C}$ and then decreases. These results meant that phosphorus in activated carbon will decompose at above $800^{\circ} \mathrm{C}$.

Based on the results from Figures 1(a) and 1(b), it is apparent that $\mathrm{MO}$ and CHMW-MO reached a markedly high weight loss at $600^{\circ} \mathrm{C}$, and the degradation rate attained the maximum at temperature near $305^{\circ} \mathrm{C}$ and $350^{\circ} \mathrm{C}$, respectively. The magnitude of weight loss was as high as $7 \mathrm{mg}$. In contrast, for the CHMW-MO-AC, the magnitude of weight loss was only below $3 \mathrm{mg}$ at temperature up to $800^{\circ} \mathrm{C}$, indicating CHMW-MO-AC has a high thermal resistance. Ioannidou and Zabaniotou [14] attributed it to the formation of higher thermally stable substances from the interaction of $\mathrm{H}_{3} \mathrm{PO}_{4}$ with carbon-containing compounds in the precursor 


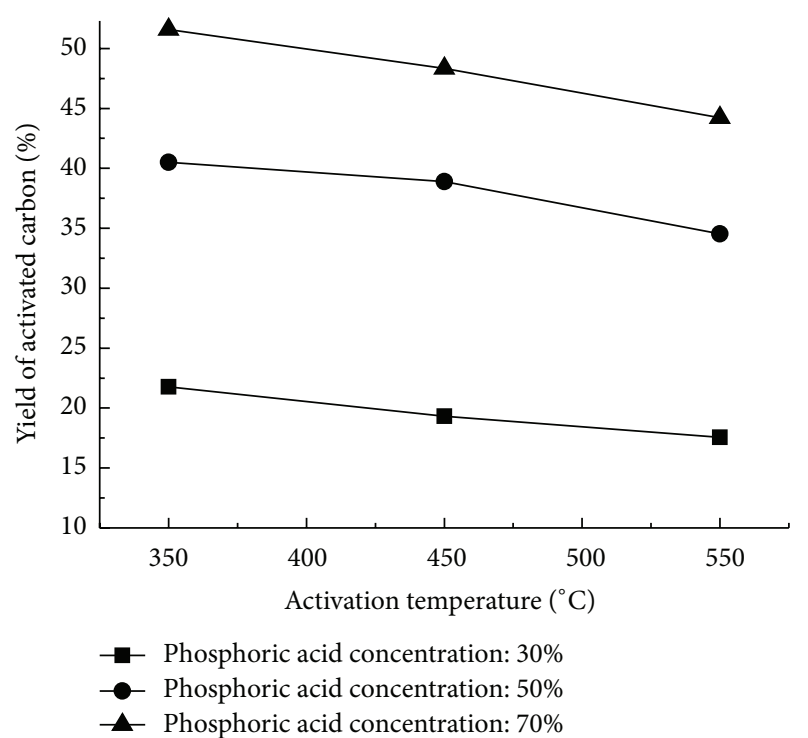

FIGURE 2: Effect of activation temperature on activated carbon yield.

materials. The detailed mechanism is not clear at present. From DTG analysis, however, it is notable that the maximum rate of weight loss for CHMW-MO-AC samples occurred in a relatively higher temperature region where virtually no weight loss for MO and CHMW-MO was recorded. This behavior is in accordance with that recently reported by Girgis and Ishak [15] and Vernersson et al. [16] for $\mathrm{H}_{3} \mathrm{PO}_{4}$ acid-impregnated cotton stalks and Arundo donax cane, respectively. Moreover, the magnitude of weight loss at 700$900^{\circ} \mathrm{C}$ increased with the increasing of impregnation ratio. Presumably, it might be volatilization of different phosphorus compounds in the CHMW-MO-ACs.

\subsection{Effect of Preparing Conditions on Yield of the Activated Carbon}

3.3.1. Effect of Activation Temperature on Yield of the Activated Carbon. The effect of activation temperature and phosphoric acid concentration on yield of activated carbon are depicted in Figure 2 as IR was 2.5 and activation time was $65 \mathrm{~min}$. Figure 2 shows that activation temperature had a great effect on the yields of activated carbons prepared from CHMW$\mathrm{MO}$ with different phosphoric acid concentrations, and the activated carbon yield was found to decrease with the increasing of temperature. This is due to the release of volatiles through pore opening and CHMW-MO gasification at high temperatures. Essentially, CHMW-MO is a natural polymer formed by cellulose, lignin, and hemicellulose. Activated at high temperature, these polymeric structures decompose and liberate most of the noncarbon elements, mainly hydrogen, oxygen, and nitrogen in the form of tars and gases, leaving the rigid carbon skeleton in the form of aromatic sheets and strips. Jagtoyen and Derbyshire [12] suggested that the reaction of lignocellulose with phosphoric acid begins as soon as the components are mixed, and first the acid attacks hemicellulose and lignin, because cellulose is more resistant

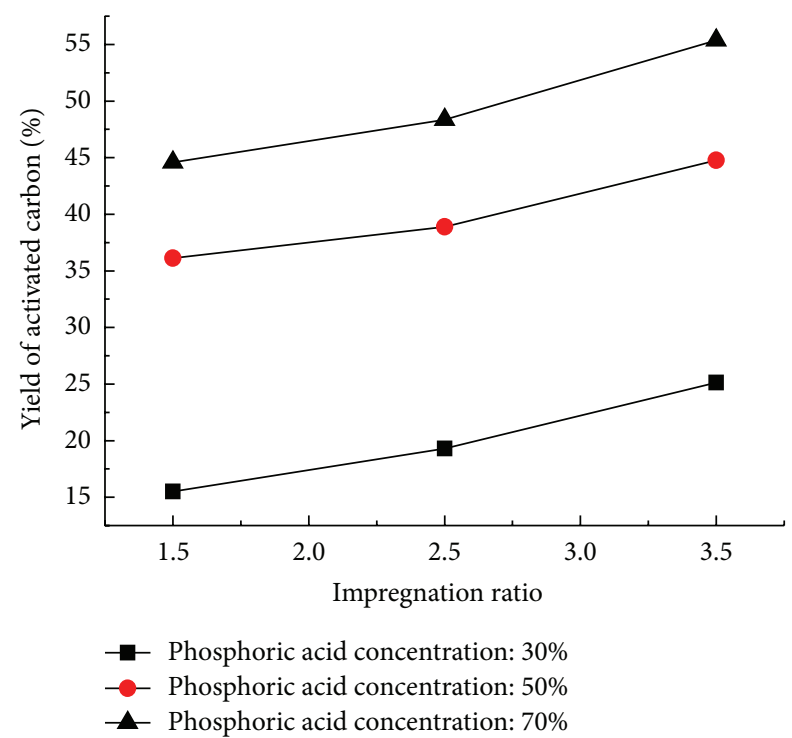

FIGURE 3: Effect of impregnation ratio on yield of activated carbon.

to acid hydrolysis, and the acid will hydrolyze glycosidic linkages in lignocellulose and cleave aryl ether bond in lignin. These reactions are accompanied by further chemical transformations including dehydration, degradation, and condensation. As the temperature increases, the aromatic condensation reactions also take place among the adjacent molecules resulting in the evolution of gaseous products from the hydroaromatic structure of carbonized char leading to the decreased yield of carbon [17].

It can be also seen that concentration of phosphoric acid had a great effect on the yields of activated carbons. The activated carbon yield is increased with the increasing of phosphoric acid concentration at different activation temperatures. As generally recognized, the transformation from CHMW-MO into activated carbon requires evolving $\mathrm{O}$ and $\mathrm{H}$ atoms as $\mathrm{H}_{2} \mathrm{O}, \mathrm{CO}, \mathrm{CO}_{2}, \mathrm{CH}_{4}$, aldehydes, or distillation of tar. The CHMW-MO-AC yield depends on the amount of carbon removed by binding with $\mathrm{O}$ and $\mathrm{H}$ atoms. Phosphoric acid can selectively stripe $\mathrm{H}$ and $\mathrm{O}$ away from $\mathrm{CHMW}$ $\mathrm{MO}$ as $\mathrm{H}_{2} \mathrm{O}$ and $\mathrm{H}_{2}$ rather than hydrocarbons, $\mathrm{CO}$, or $\mathrm{CO}_{2}$ [18]. As expected, phosphoric acid activation increases the carbon content and decreases hydrogen and oxygen content. The higher the phosphoric acid concentration, the lower the oxygen and hydrogen content, and the higher the carbon content. Activated carbon yield increases rapidly when concentration of phosphoric acid aqueous solution is less than $50 \%$, but when the concentration is higher than $50 \%$, the yield increases gently. So, an economic evaluation on maximum yield of activated carbon and least consumption on phosphoric acid must be done. In all subsequent experiments, activation temperature was selected at $550^{\circ} \mathrm{C}$.

3.3.2. Effect of Impregnation Ratio on Yield of the Activated Carbon. Impregnation ratio is another critical parameter affecting the activated carbon yield. Figure 3 shows the activated carbon yield prepared at different impregnation 


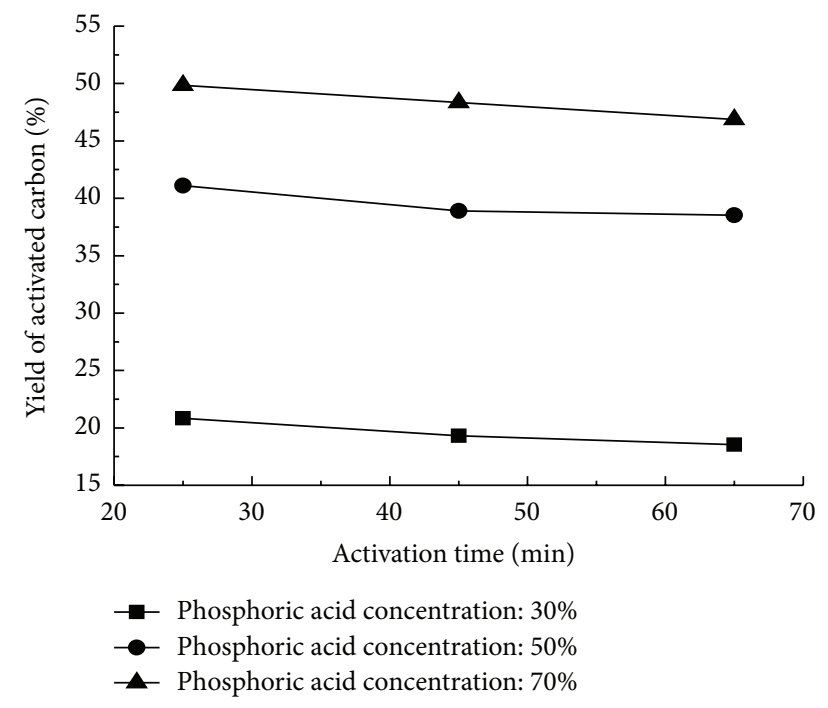

FIGURE 4: Effect of activation time on yield of activated carbon.

ratios as the activation time and activation temperature were $65 \mathrm{~min}$ and $550^{\circ} \mathrm{C}$, respectively. From Figure 3, it is obvious that the activated carbon yield is increased with the increasing of impregnation ratio. The behavior might be explained using mechanism of phosphoric acid activation [12]. Phosphoric acid acts as catalyst on the one hand promoting bond cleavage reactions, on the other hand facilitating crosslinking via cyclization, condensation, and being able to combine with organic species to form phosphate linkages, such as phosphate and polyphosphate esters. The more the phosphoric acid was used, the larger the amounts of polyphosphates are formed and retained in activated carbon in the form of insoluble metal phosphates, or physically entrapped, even though phosphoric acid is removed after reaction by leaching with ample usage of fresh water to recover the reagent. Using techniques such as FTIR, XPS, EDX, and NMR, Jagtoyen and Derbyshire [12] and Puziy et al. [13, 19, 20] confirmed the presence of phosphorous after $\mathrm{H}_{3} \mathrm{PO}_{4}$ activation, and the maximum content of phosphate was as high as $9 \%$.

3.3.3. Effect of Activation Time on Yield of the Activated Carbon. As indicated in Figure 4, it is obvious that yield of activated carbon decreased as the activation time increased. The reason is that carbon burning-off and tar volatilization become more severe due to longer activation time at higher temperature. The same result was also observed by other researchers $[14,16,17]$. Moreover, a shorter activation time can reduce the energy consumption and improve economy of the production process and should be accepted in preparing activated carbon.

3.4. $\mathrm{N}_{2}$ Adsorption-Desorption Isotherms of the CHMW$M O-A C s$. Figure 5(a) illustrates $\mathrm{N}_{2}$ adsorption-desorption isotherms (at $77 \mathrm{~K}$ ) of $\mathrm{MO}$ and CHMW-MO. The low $\mathrm{N}_{2}$ adsorbed amount for $\mathrm{MO}$ was observed, indicating $\mathrm{MO}$ is less of porous structure. When MO became CHMWMO by decoction, some inorganic matters and part of water-soluble organic and inorganic matters were removed; both behaviors made porous structure of CHMW-MO open and increased porosity and the adsorbed $\mathrm{N}_{2}$ amount at relatively high pressures.

Some researchers suggested that the activation temperature has a significant influence on physical structure characterization of the resultant activated carbon [21, 22]. So, three activated carbon samples prepared at different activation temperature were performed by nitrogen adsorption at $77 \mathrm{~K}$ in the relative pressure range of $10^{-6}$ to $1 \mathrm{~atm}$.

Figure 5(b) indicates $\mathrm{N}_{2}$ adsorption-desorption isotherms of three samples produced at $50 \% \quad \mathrm{H}_{3} \mathrm{PO}_{4}$ concentration, impregnation ratio of 2.5 , activation time of $65 \mathrm{~min}$, and three different activation temperatures ranging from 350 to $550^{\circ} \mathrm{C}$ (denoted by S-350, S-450, and $\mathrm{S}-550$, resp.). Their isotherms show a transition from type I to type IV, according to the IUPAC classification [23]. S-350 and S-450 are the isotherms of type I that do not display an apparent desorption hysteresis loop; nitrogen adsorption capacity rises sharply at low relative pressures and then reaches a plateau which is parallel to the relative pressure axis. This isotherm occurs due to an increase in the interaction between the solid surface and the gas molecules in micropores which lead to an increasement in adsorption, especially at low relative pressure. The adsorption behaviors of S-350 and S-450 indicate that they are highly microporous materials. When the activation temperature rises over $450^{\circ} \mathrm{C}$, a noticeable variation in the shape of the isotherm can be observed. The isotherm becomes a combination of type I and type IV isotherms. The initial part of the isotherms of S-550 is of type I with significant nitrogen adsorption capacity at low relative pressures, which is characteristic of adsorption in micropores. At intermediate and high relative pressures, the isotherm shows a broad knee. As the relative pressure increases to 0.4 , the isotherm exhibits type IV with a distinct desorption hysteresis loop (H4 type). The hysteresis loop is usually associated with monolayermultilayer adsorption followed by capillary condensation in mesoporous structures [19]. It means that the dominant pores in S-550 are mesopores. The results indicate that the activated carbons obtained from three various activation temperatures show both microporous and mesoporous structures.

As indicated in Figure 5, nitrogen adsorption capacity of the three samples increased with increasing of activation temperature. The facts indicate that, with the increasing of activation temperature, the existing pores are widened, and the new pores are created continuously. This is likely caused by the removal of phosphorous species from the activated mixture due to the rigorous reactions between phosphoric acid and CHMW-MO at higher activation temperature or destruction of wall between adjacent micropores by reaction with phosphoric acid. Compared with the relative nitrogen uptake of sample from temperature above $450^{\circ} \mathrm{C}$, the relative nitrogen uptake of samples prepared at temperatures between 350 and $450^{\circ} \mathrm{C}$ increases quickly, meaning that the development of surface area and porous texture of samples prepared at temperature below $450^{\circ} \mathrm{C}$ are significant. But when the temperature is above $450^{\circ} \mathrm{C}$, the development is slow. This fact 


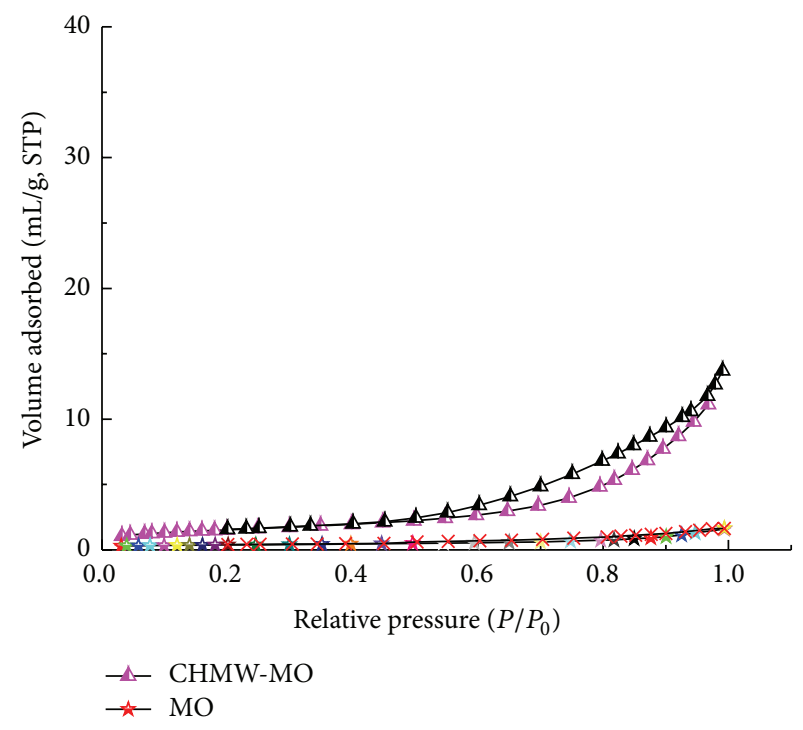

(a)

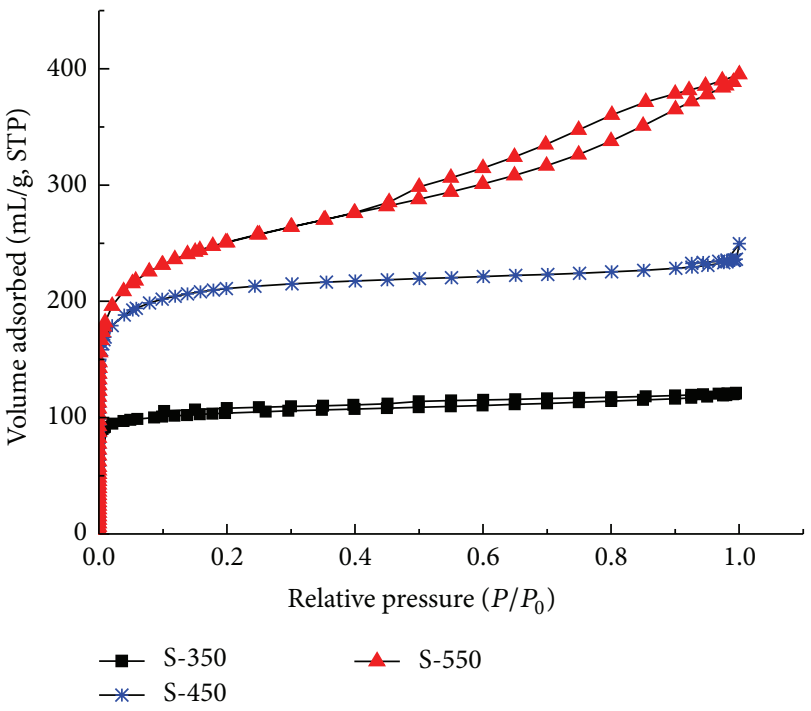

(b)

FIGURE 5: Nitrogen adsorption-desorption isotherms of MO, CHMW-MO, and its activated carbons. (a) MO and CHMW-MO samples; (b) CHMW-MO-AC samples prepared at different activation temperatures.

TABLE 3: $S_{\text {BET }}$ and porous texture of MO, CHMW-MO, and its activated carbons.

\begin{tabular}{lcccccc}
\hline & $\begin{array}{c}S_{\mathrm{BET}} \\
\left(\mathrm{m}^{2} / \mathrm{g}\right)\end{array}$ & $\begin{array}{c}S_{\text {micro }} \\
\left(\mathrm{m}^{2} / \mathrm{g}\right)\end{array}$ & $\begin{array}{c}V_{\text {micro }} \\
\left(\mathrm{cm}^{3} / \mathrm{g}\right)\end{array}$ & $\begin{array}{c}V_{\text {meso }} \\
\left(\mathrm{cm}^{3} / \mathrm{g}\right)\end{array}$ & $\begin{array}{c}V_{\text {total }} \\
\left(\mathrm{cm}^{3} / \mathrm{g}\right)\end{array}$ & $\begin{array}{c}D_{p} \\
\left(\mathrm{~nm}^{2}\right)\end{array}$ \\
\hline MO & 0.453 & 0.5725 & 0.0003 & 0.0010 & 0.0013 \\
CHMW-MO & 27.33 & 28.13 & 0.0077 & 0.0536 & 0.0613 \\
S-350 & 406.49 & 380.17 & 0.1569 & 0.05 & 0.2069 & 0.75 .18 \\
S-450 & 794.50 & 765.02 & 0.3205 & 0.1075 & 0.428 \\
S-550 & 920.33 & 704.20 & 0.3054 & 0.3971 & 0.7025 \\
\hline
\end{tabular}

is indicative of an increase in the specific surface area $\left(S_{\mathrm{BET}}\right)$ following the same trend. The values $S_{\mathrm{BET}}$ as well as the microand mesopore volumes ( $V_{\mathrm{mi}}$ and $V_{\mathrm{me}}$, resp.) were summarized in Table 3 to corroborate this conclusion.

Based on adsorption isotherms, BET surface area and porous texture of MO, CHMW-MO, and its activated carbons are given in Table 3. Three activated carbons show a well-developed porous structure with BET surface area up to $920 \mathrm{~m}^{2} / \mathrm{g}$ and total pore volume up to $0.7025 \mathrm{~cm}^{3} / \mathrm{g}$ (for S-550). BET surface area and all pore volumes (total, micropore, and mesopore) are increased with the increasing of activation temperature. As temperature increased from 350 to $450^{\circ} \mathrm{C}$, BET surface area and micropore surface area of sample obtained at temperature of $450^{\circ} \mathrm{C}$ almost doubled for sample of temperature $350^{\circ} \mathrm{C}$. Similarly, all pore volumes (total, micropore, and mesopore) are corresponding to similar increasement of BET surface area. But the ratio of micropores to the total porosity in the two activated carbons almost remains a constant which is $75.8 \%$ and $75.4 \%$, respectively. However, activation temperature increased from 450 to $550^{\circ} \mathrm{C}$; increased values of surface area in sample are relatively less, but micropore area and micropore volume decreased marginally; the ratio of micropore to the total porosity decreased from $75.4 \%$ to $43.7 \%$. The mesopore volume is only $0.1075 \mathrm{~mL} / \mathrm{g}$ for S- 450 obtained at $450^{\circ} \mathrm{C}$, but the mesopore volume of S-550 obtained at $500^{\circ} \mathrm{C}$ more appreciably increased to $0.3971 \mathrm{~mL} / \mathrm{g}$; as activation temperature is raised to $550^{\circ} \mathrm{C}$, the ratio of mesopore to the total porosity increased to $56.5 \%$. There is no indication that the mesopore volume has attained a maximum at the highest temperature used here. The facts suggest that activation temperature is less than $450^{\circ} \mathrm{C}$, and both micropore and mesopore are continuously created at a constant relative rate. When the activation temperature is over $450^{\circ} \mathrm{C}$, the contribution of mesopore to the total porosity increases suggesting pore widening exceeds pore creation. These results confirm that activation temperature is an important variable, and it can be used to change the pore size distribution in activated carbon. $450^{\circ} \mathrm{C}$ is a critical temperature for adjusting the ratio of micropores to mesopores in preparing activated carbon from $\mathrm{CHMW}-\mathrm{MO}$ by phosphoric acid activation.

3.5. Micro- and Mesopore Structure Characteristics of the Activated Carbons. The structural heterogeneity of porous material is generally characterized in terms of the pore size distribution. Using the Barret-Joyner-Halenda (BJH) method, the pore size distributions of three CHMW-MOACs prepared at different activation temperatures were 


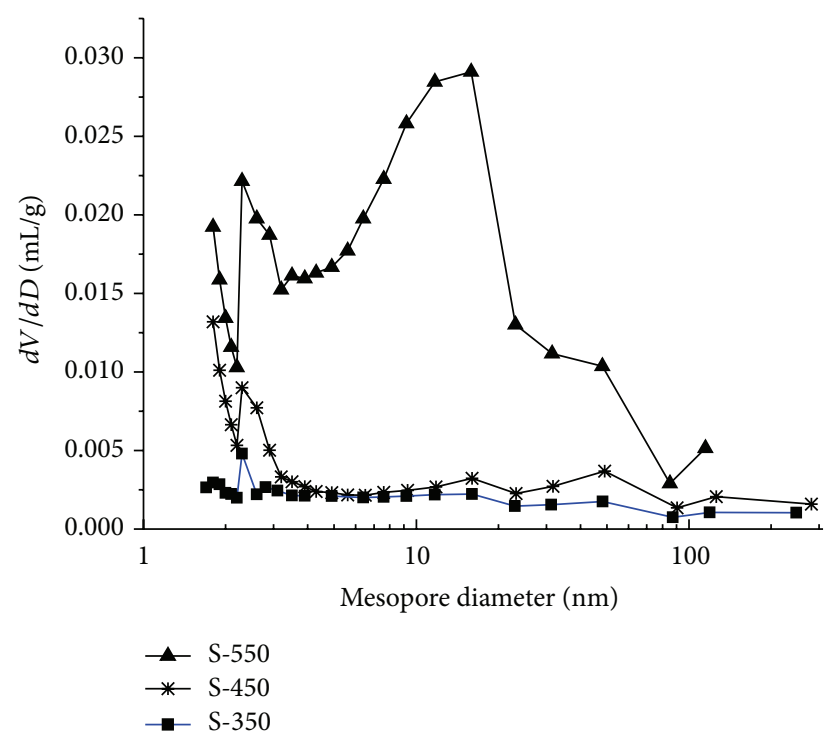

Figure 6: BJH pore size distribution in CHMW-MO-ACs.

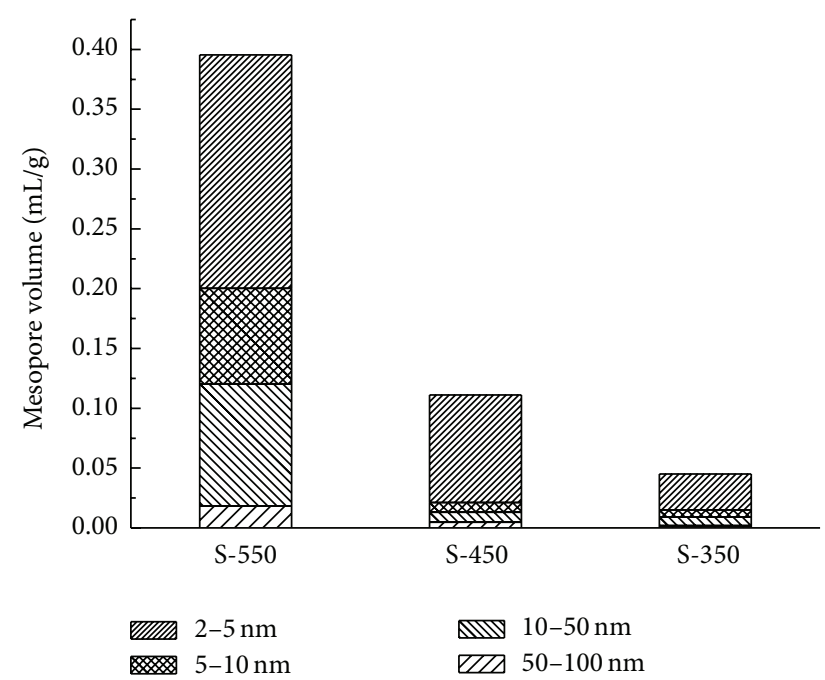

FIGURE 7: Comparison of mesopore porosity development in CHMW-MO-ACs.

obtained and showed in Figure 6. Samples S-350 and S-450 show a very similar mesopore size distribution. For sample S-350, BJH pore size distribution keeps nearly unchanged at overall pore size from 2 to $300 \mathrm{~nm}$, except a weak peak showed at a pore diameter of about $2.5 \mathrm{~nm}$. A narrow peak of the activated carbon S- 450 approaches $2.8 \mathrm{~nm}$. For sample S-550, there are bimodal distribution. The first peak position shifts to $3.0 \mathrm{~nm}$ and a highest peak with maximum pore size appears at $11 \mathrm{~nm}$. The shift of peak position mainly comes from the development of the mesoporosity. The $\mathrm{BJH}$ average pore diameter of S-350, S-450, and S-550 is 3.43, 2.91, and $4.03 \mathrm{~nm}$, respectively.

Figure 7 shows mesopore cumulative volume of three $\mathrm{CHMW}-\mathrm{MO}-\mathrm{ACs}$ at four different pore ranges. It is illustrated that activated carbon S-550 has abundant mesoporosity. Mesopore volume of S-550 was the largest compared to

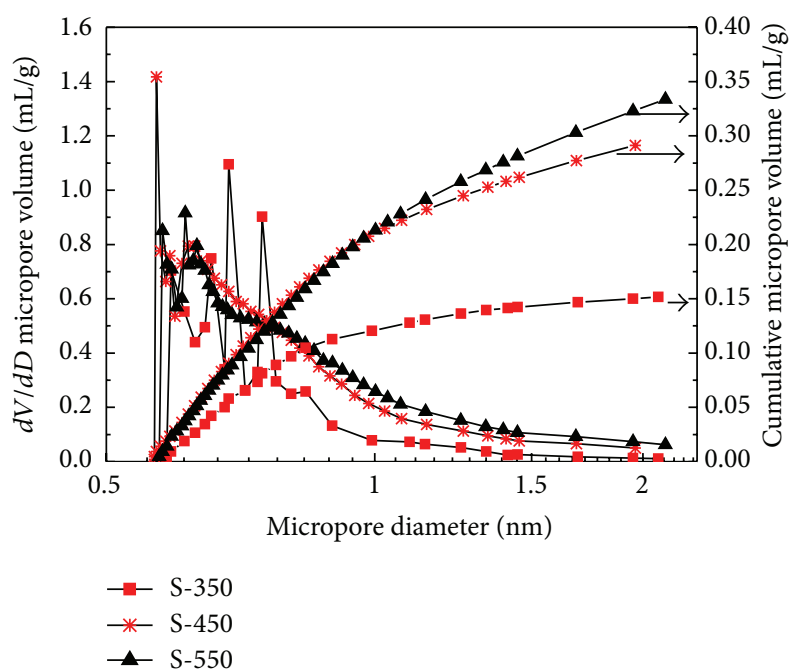

Figure 8: Micropore size distribution in CHMW-MO-ACs from HK method.

that of the activated carbons S-350 and S-450. Particularly, $\mathrm{S}-550$ had a relatively large mesopore volume in the range of $2-5 \mathrm{~nm}$. The activation reaction is relatively moderate at temperature less than $450^{\circ} \mathrm{C}$, resulting in relatively narrow $\mathrm{BJH}$ size distribution. The reaction became rigorous at $550^{\circ} \mathrm{C}$, and some micropores are converted to mesopores and new mesopores are developed. Subsequently, the pore volume is higher. With the increasing of activation temperature, the increased value of pores between 2 and $5 \mathrm{~nm}$ is the largest, but the growth rate of pores between 10 and $50 \mathrm{~nm}$ attains maximum. This indicates that, with the increasing of activation temperature, many micropores are enlarged and eventually become mesoporous.

For understanding the microporous structure of the CHMW-MO-ACs in a more detailed manner, the $\mathrm{N}_{2}$ adsorption data was analyzed by the Horvath-Kawazoe (HK) method. Micropore size distributions in three CHMW-MOACs are shown in Figure 8. The HK plot of S-350 is bimodal, with two maxima centered on 0.65 and $0.75 \mathrm{~nm}$. Similarly, this behavior is also observed in S-450 and S-550, regardless of what the activation temperature is. Nevertheless, S-450 and S-550 have larger values of the micropore volume, because the area under the HK curve is larger. Compared to the other two samples, the S-450 has the largest micropore volume around $0.55 \mathrm{~nm}$, meaning more micropores are opened at activation temperature of $450^{\circ} \mathrm{C}$. With the increasing of activation temperature from 350 to $450^{\circ} \mathrm{C}$, the increasing of the micropore volume indicated that more new micropores were created, and original micropore widened simultaneously. However, as activation temperature is higher than $450^{\circ} \mathrm{C}$, the volume of the micropores size $(<0.9 \mathrm{~nm})$ decreases slightly and volume of the micropores size $(>0.9 \mathrm{~nm})$ increases, meaning pore widening only occurs in lager micropore range $(>0.9 \mathrm{~nm})$. Generally, with the increasing of activation temperature, micropore widening should occur above both micropore size ranges. The behavior probably resulted from the pretreatment 


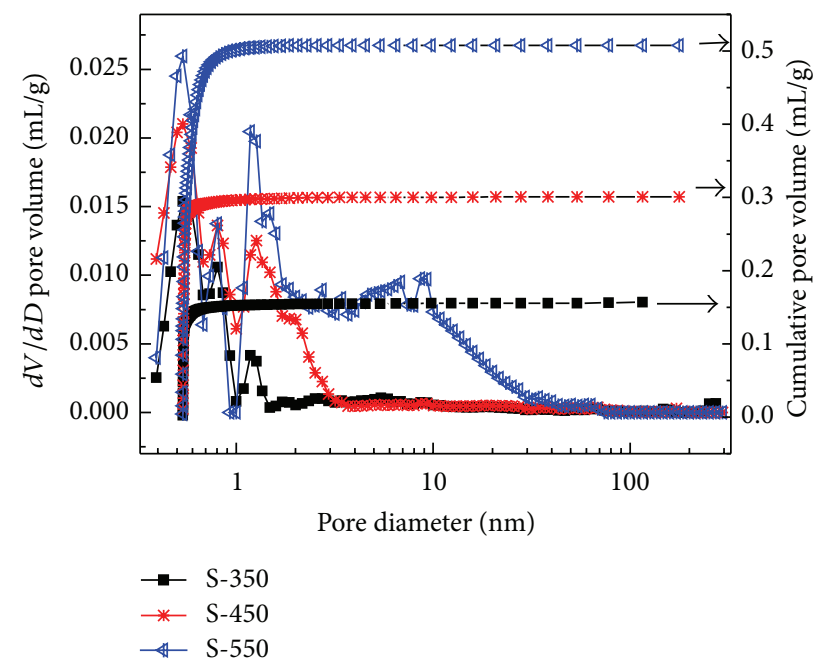

Figure 9: Pore size distribution of CHMW-MO-ACs by DFT method.

of CHMW-MO-ACs, but more details need to be further researched.

For clarity, pore size distribution by applying the density functional theory (DFT) to $\mathrm{N}_{2}$ adsorption data is shown in Figure 9. Three strong peaks could be observed for the three CHMW-MO-ACs. The S-350 has analogous pore size distribution pattern to $\mathrm{S}-450$ that is multimodal pattern with distinct maxima in the micropore regions. For ultramicropores $(<0.7 \mathrm{~nm})$, the three samples have a strong peak around $0.65 \mathrm{~nm}$; however the peak magnitude increases with activation temperature. In the micropore region, two different maxima at 0.8 and $1.3 \mathrm{~nm}$ and a minimum at $1 \mathrm{~nm}$ could be observed; the minimum is attributed to modeling assumptions in DFT method. The peak at $0.8 \mathrm{~nm}$ keeps unchanged for three samples, but at $1.3 \mathrm{~nm}$, the peak has a tendency to shift to large micropore region and peak magnitude increases with activation temperature. There are no maxima in mesopore/macropore for S-350 and S-450, with the only exception of S-550, in which three weak peaks are observed at 3,7 , and $10 \mathrm{~nm}$, respectively.

3.6. SEM Analysis of S-550. The interior structure of activated carbon determines its adsorption capacity. SEM of surface morphology of activated carbon could give qualitative analysis for interior structure of activated carbon. SEM images of the surface morphology of S-550 are given in Figure 10. Figure 10 shows that the external surface of S-550 has irregular, heterogeneous surface characteristics, cracks, or pores and some grains in various micron sizes exist on its external surface. From the above observations, it can be concluded that more complicated topology exists inside S550 .

\section{Conclusions}

From the results obtained above, the following conclusions may be drawn:
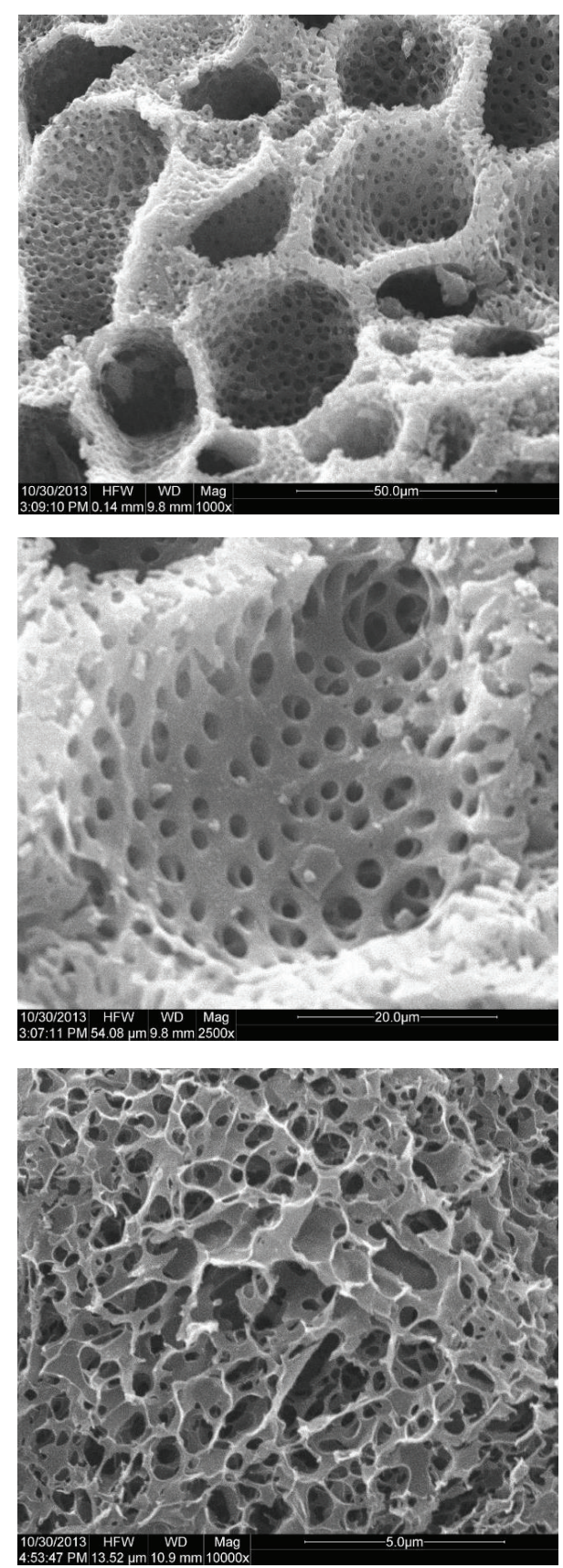

Figure 10: SEM images of activated carbon S-550.

(i) CHMW-MO can be used as precursor for the preparation of activated carbon.

(ii) The preparation conditions influence yield of the CHMW-MO-ACs. So, for improving the economy of process and the final properties of the activated carbons, preparation conditions should be carefully controlled.

(iii) CHMW-MO-ACs with higher surface area, $920 \mathrm{~m}^{2} / \mathrm{g}$, and total pore volume, $0.703 \mathrm{~cm}^{3} / \mathrm{g}$, can be prepared from CHMW-MO at $50 \% \mathrm{H}_{3} \mathrm{PO}_{4}$ concentration, impregnation ratio of 2.5, activation time of $65 \mathrm{~min}$, 
and temperatures of $550^{\circ} \mathrm{C}$. Activation temperature strongly affects porous texture of the CHMW-MOACs. A threshold activation temperature exists for the development of mesopore in CHMW-MO-ACs. The reason may be due to the unique properties of CHMW preprepared by decoction or percolation.

\section{Conflict of Interests}

The authors declare that there is no conflict of interests regarding the publication of this paper.

\section{Acknowledgments}

The authors gratefully acknowledge financial support of the Science \& Technology Project of Wuhan city (2013061001010481), the Natural Science Foundation of China (U1261204), and the Foundation of State Key Laboratory of Coal Combustion (FSKLCCA1507).

\section{References}

[1] A. Keshtkar, H. Ghaforian, G. Abolhamd, and B. Meyssami, "Dynamic simulation of cyclic batch anaerobic digestion of cattle manure," Bioresource Technology, vol. 80, no. 1, pp. 9-17, 2001.

[2] A. Dą̧rowski, P. Podkościelny, Z. Hubicki, and M. Barczak, "Adsorption of phenolic compounds by activated carbon-a critical review," Chemosphere, vol. 58, no. 8, pp. 1049-1070, 2005.

[3] V. Gómez-Serrano, E. M. Cuerda-Correa, M. C. FernándezGonzález, M. F. Alexandre-Franco, and A. Macías-García, "Preparation of activated carbons from chestnut wood by phosphoric acid-chemical activation. Study of microporosity and fractal dimension," Materials Letters, vol. 59, no. 7, pp. 846-853, 2005.

[4] A. L. Cukierman, P. A. Della Rocca, P. R. Bonelli, and M. C. Cassanello, "On the study of thermochemical biomass conversion," Trends in Chemical Engineering-Research Trends, vol. 3, pp. 129-144, 1996.

[5] M. J. Antal Jr., "Biomass pyrolysis: a review of the literature," in Advances in Solar Energy, K. W. Boer and J. A. Duffie, Eds., vol. 2, pp. 175-255, American Solar Energy Society, Boulder, Colo, USA, 1985.

[6] A. M. M. Vargas, A. L. Cazetta, C. A. Garcia et al., "Preparation and characterization of activated carbon from a new raw lignocellulosic material: flamboyant (Delonix regia) pods," Journal of Environmental Management, vol. 92, no. 1, pp. 178-184, 2011.

[7] T.-H. Liou, "Development of mesoporous structure and high adsorption capacity of biomass-based activated carbon by phosphoric acid and zinc chloride activation," Chemical Engineering Journal, vol. 158, no. 2, pp. 129-142, 2010.

[8] B. R. Puri, "Surface complexes on carbons," in Chemistry and Physics of Carbon, P. L. Walker Jr., Ed., vol. 6, pp. 191-282, Marcel Dekker, New York, NY, USA, 1970.

[9] G. de la Puente, J. J. Pis, J. A. Menéndez, and P. Grange, “Thermal stability of oxygenated functions in activated carbons," Journal of Analytical and Applied Pyrolysis, vol. 43, no. 2, pp. 125-138, 1997.

[10] F. Suárez-García, A. Martínez-Alonso, and J. M. D. Tascón, "Activated carbon fibers from Nomex by chemical activation with phosphoric acid," Carbon, vol. 42, no. 8-9, pp. 1419-1426, 2004.

[11] Ö. Tunç, H. Tanaci, and Z. Aksu, "Potential use of cotton plant wastes for the removal of Remazol Black B reactive dye," Journal of Hazardous Materials, vol. 163, no. 1, pp. 187-198, 2009.

[12] M. Jagtoyen and F. Derbyshire, "Activated carbons from yellow poplar and white oak by $\mathrm{H}_{3} \mathrm{PO}_{4}$ activation," Carbon, vol. 36, no. 7-8, pp. 1085-1097, 1998.

[13] A. M. Puziy, O. I. Poddubnaya, A. Martínez-Alonso, F. SuárezGarcía, and J. M. D. Tascón, "Synthetic carbons activated with phosphoric-acid I. Surface chemistry and ion binding properties," Carbon, vol. 40, no. 9, pp. 1493-1505, 2002.

[14] O. Ioannidou and A. Zabaniotou, "Agricultural residues as precursors for activated carbon production: a review," Renewable and Sustainable Energy Reviews, vol. 11, no. 9, pp. 1966-2005, 2007.

[15] B. S. Girgis and M. F. Ishak, "Activated carbon from cotton stalks by impregnation with phosphoric acid," Materials Letters, vol. 39, no. 2, pp. 107-114, 1999.

[16] T. Vernersson, P. R. Bonelli, E. G. Cerrella, and A. L. Cukierman, "Arundo donax cane as a precursor for activated carbons preparation by phosphoric acid activation," Bioresource Technology, vol. 83, no. 2, pp. 95-104, 2002.

[17] S. Timur, I. C. Kantarli, E. Ikizoglu, and J. Yanik, "Preparation of activated carbons from Oreganum stalks by chemical activation," Energy and Fuels, vol. 20, no. 6, pp. 2636-2641, 2006.

[18] F. Caturla, M. Molina-Sabio, and F. Rodríguez-Reinoso, "Preparation of activated carbon by chemical activation with $\mathrm{ZnCl}_{2}$," Carbon, vol. 29, no. 7, pp. 999-1007, 1991.

[19] A. M. Puziy, O. I. Poddubnaya, R. P. Socha, J. Gurgul, and M. Wisniewski, "XPS and NMR studies of phosphoric acid activated carbons," Carbon, vol. 46, no. 15, pp. 2113-2123, 2008.

[20] N. V. Sych, S. I. Trofymenko, O. I. Poddubnaya et al., "Porous structure and surface chemistry of phosphoric acid activated carbon from corncob," Applied Surface Science, vol. 261, pp. 7582, 2012.

[21] R. C. Bansal, J. B. Donnet, and F. Stoeckli, Active Carbon, Marcel Dekker, New York, NY, USA, 1988.

[22] F. Rouquerol, J. Rouquerol, and K. S. Sing, Adsorption by Powders and Porous Solids, Principles, Methodology and Applications, Academic Press, London, UK, 1999.

[23] K. S. W. Sing, D. H. Everett, R. A. W. Haul et al., "Reporting physisorption data for gas/solid systems with special reference to the determination of surface area and porosity," Pure and Applied Chemistry, vol. 57, pp. 603-609, 1985. 

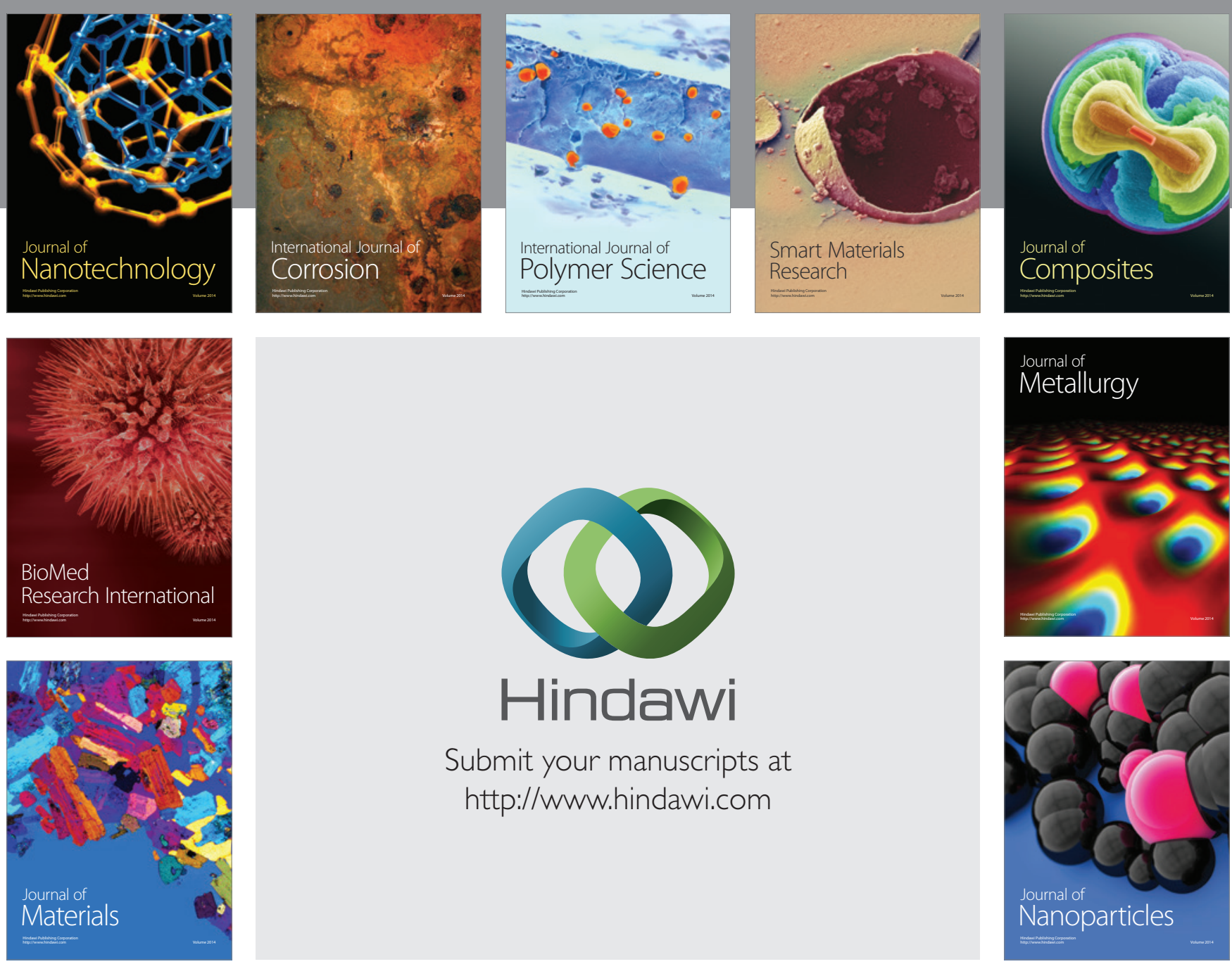

Submit your manuscripts at http://www.hindawi.com
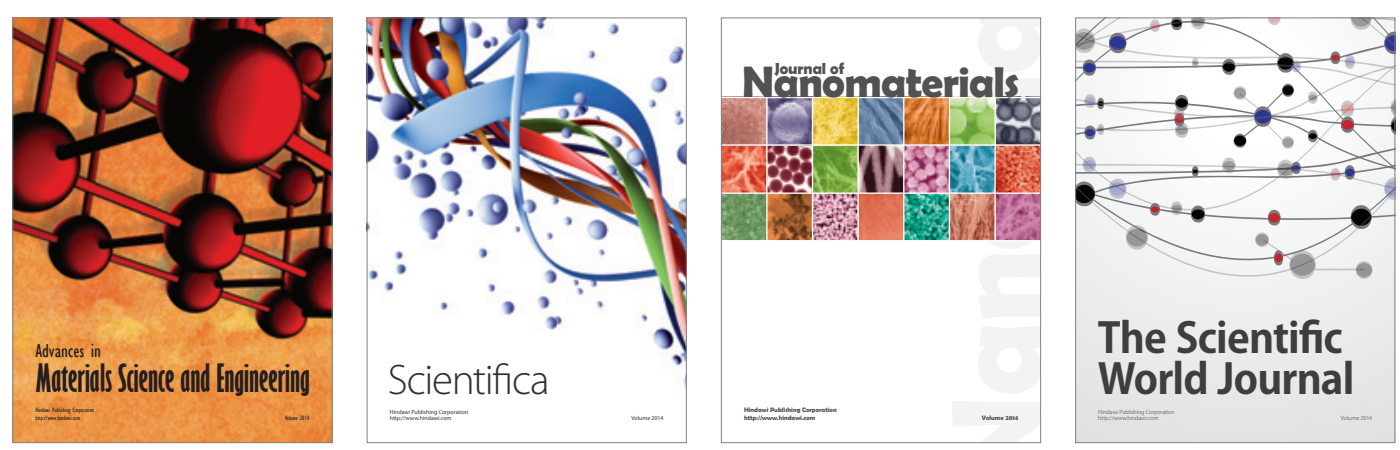

\section{The Scientific World Journal}
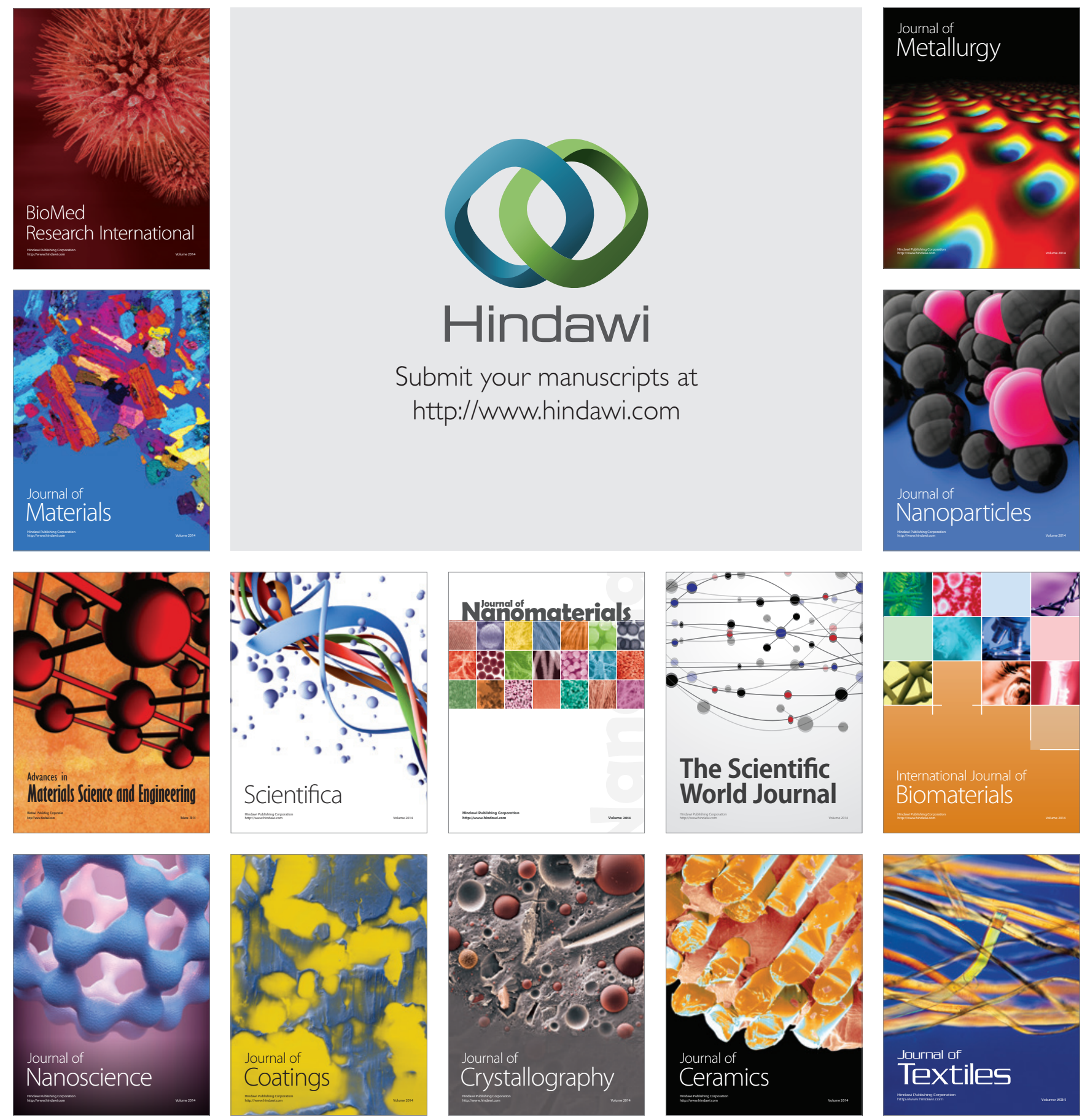